\title{
Politique
}

Politique

\section{Politique de la science au Québec et autonomie du champ scientifique}

\section{Michel Trépanier}

Numéro 22, automne 1992

URI : https://id.erudit.org/iderudit/040731ar

DOI : https://doi.org/10.7202/040731ar

Aller au sommaire du numéro

Éditeur(s)

Société québécoise de science politique

ISSN

0711-608X (imprimé)

1918-6584 (numérique)

Découvrir la revue

Citer cet article

Trépanier, M. (1992). Politique de la science au Québec et autonomie du champ scientifique. Politique, (22), 101-135. https://doi.org/10.7202/040731ar
Résumé de l'article

Depuis la fin des années 1970, l'État québécois a déployé beaucoup d'efforts pour identifier et choisir, dans le cadre de sa politique scientifique et technologique, des domaines prioritaires dans lesquels concentrer ses dépenses de R-D. L'analyse de ces tentatives montre que le gouvernement du Québec a la plupart du temps fonctionné en vase clos sans vraiment impliquer, dans la prise de décision et le choix des domaines prioritaires, les représentants des universités et de l'entreprise. Interventionniste, l'État québécois s'est doté d'un appareil de politique scientifique et, de concert avec lui, a créé de nouveaux centres de recherche et mis sur pied de nouveaux programmes de soutien à la R-D. Toutefois, ces interventions n'ont pas été assez nombreuses et extensives pour réduire significativement l'autonomie du champ scientifique; les chercheurs sont en bonne partie demeurés libres d'orienter leurs travaux en fonction des enjeux et des intérêts propres à leur champ. 


\title{
POLITIQUE DE LA SCIENCE AU QUÉBEC ET AUTONOMIE DU CHAMP SCIENTIFIQUE ·
}

\author{
Michel Trépanier \\ INRS-Urbanisation
}

\begin{abstract}
Depuis la fin des années 1970, l'État québécois a déployé beaucoup d'efforts pour identifier et choisir, dans le cadre de sa politique scientifique et technologique, des domaines prioritaires dans lesquels concentrer ses dépenses de R-D. L'analyse de ces tentatives montre que le gouvernement du Québec a la plupart du temps fonctionné en vase clos sans vraiment impliquer, dans la prise de décision et le choix des domaines prioritaires, les représentants des universités et de l'entreprise. Interventionniste, l'État québécois s'est doté d'un appareil de politique scientifique et, de concert avec lui, a créé de nouveaux centres de recherche et mis sur pied de nouveaux programmes de soutien à la R-D. Toutefois, ces interventions n'ont pas été assez nombreuses et extensives pour réduire significativement I'autonomie du champ scientifique; les chercheurs sont en bonne partie demeurés libres d'orienter leurs travaux en fonction des enjeux et des intérêts propres à leur champ.
\end{abstract}

Dans la plupart des pays industrialisés, la mondialisation des marchés et le rôle accru de la science et de la technologie' dans le développement de l'industrie manufacturière et des services ont incité les gouvernements à mobiliser plus efficacement la science

- Certaines des idées de ce texte étaient déjà présentes dans un document que j'ai préparé pour le Conseil de la Science et de la Technologie en 1991 : Mécanismes d'identification de secteurs prioritaires en matière de $R-D$. Je remercie le Conseil de $\mathrm{m}^{\prime}$ avoir autorisé à les réutiliser ici. Je tiens à remercier Yves Gingras et Maurice l'Abbé pour leurs commentaires et suggestions. Je remercie également les évaluateurs pour leurs commentaires judicieux. Merci, enfin, a Daniel Latouche pour $m^{\prime}$ avoir encouragé à soumettre cet article à la Revue québécoise de science politique.

1 En raison de leur emploi très répandu, les termes "technologie» et «technologique» reviendront souvent au cours de l'article à la place de "techniquen.

Revue québécoise de science politique, no 22, automne 1992. 
et la technologie à des fins de développement économique. On remarque en effet que, depuis quelques années, la majorité des stratégies de développement économique, qu'elles soient nationales ou régionales, font de la science et de la technologie un outil d'intervention privilégié. Combiné à une limitation des ressources financières et à la multiplication des possibilités scientifiques et technologiques, ce nouvel objectif de développement économique a entraîné une révision des stratégies d'intervention et de développement en matière de science et de technologie et a conduit au choix de domaines prioritaires dans lesquels concentrer les ressources.

Confrontée à ces réalités, la ministre de l'Enseignement supérieur et de la Science du Québec a demandé au Conseil de la science et de la technologie de préparer un avis sur les enjeux et les priorités du développement scientifique et technologique du Québec $^{2}$. Selon la ministre, c'est la recherche de la meilleure utilisation possible de la science et de la technologie à des fins de développement économique qui devait servir de guide aux travaux du Conseil. Et pour bien marquer cette orientation, c'est en concertation avec le ministère de l'Industrie, du Commerce et de la Technologie qu'elle lui adressait cette demande.

En regard du contexte évoqué plus haut, la demande de la ministre semble tout à fait appropriée et paraît s'inscrire dans une conjoncture relativement récente, qui donne à l'exercice un air de nouveauté. Pourtant, un examen rapide de l'histoire de la politique scientifique et technologique du Québec nous montre que plusieurs exercices de ce type ont déjá été effectués et que la question des priorités scientifiques et technologiques, et surtout celle des priorités sectorielles, est à l'ordre du jour depuis près de vingt ans. Nous ne savons pourtant que très peu de choses sur les tentatives de réponses qui ont été faites. Aussi, apparaît-il important, au moment même où le Conseil de la science et de la technologie rend publique sa réflexion sur la mise en œuvre d'un processus permanent de choix des priorités scientifiques et technologiques,

2 Ministère de l'Enseignement supérieur et de la Science, Cabinet de la ministre, «Enjeux et priorités du développement scientifique et technologique du Québec», communiqué du 14 avril 1992. Document cité en annexe de l'ouvrage suivant: Conseil de la Science et de la Technologie, Urgence technologie. Pour un Québec audacieux, competitif et prospère, Sainte-Foy, avril 1993. 
d'analyser le déroulement des tentatives passées et d'en évaluer les forces et les faiblesses.

\section{La négociation du degré d'autonomie du champ scientifique}

Pour comprendre les caractéristiques et les orientations des exercices de choix de priorités ainsi que leurs effets sur l'activité de recherche et développement (R-D), il faut d'abord s'arrêter aux éléments spécifiques de l'espace social auquel ils s'adressent, en l'occurrence le champ scientifique. Le champ scientifique est défini comme "l'espace de jeu d'une lutte de concurrence ayant pour enjeu spécifique le monopole de l'autorité scientifique inséparablement définie comme capacité technique et comme pouvoir social ${ }^{3}$ ". Il a ceci de particulier que les producteurs de savoir tendent à n'avoir d'autres clients possibles que leurs concurrents. Ce n'est donc le plus souvent que d'un pair concurrent que peut venir la reconnaissance scientifique qui donne à son détenteur le type particulier de capital dont il a besoin pour maintenir ou améliorer sa position dans le champ ${ }^{4}$.

II en va ainsi pour trois raisons. D'abord, parce que le niveau et les caractéristiques des connaissances nécessaires pour jouer le jeu sont tels que seuls les joueurs-chercheurs peuvent s'approprier le travail scientifique et en évaluer les mérites. Ensuite, parce que ce sont essentiellement les joueurs-chercheurs qui contrôlent la transmission des connaissances requises et l'admission des nouveaux entrants. Et, finalement, parce que le recours à une autorité extérieure pour faire reconnaître les mérites d'un travail attire le discrédit ${ }^{5}$.

3 Bourdieu, P. (1975), "La spécificité du champ scientifique et les conditions sociales du progrès de la raison", in Sociologie et sociétés, vol. 7, no 1, p. 91-92.

4 Bourdieu, P. (1975), op. cit., p. 95.

5 Bourdieu, P. (1975), op. cit., p. 95. L'histoire contemporaine des sciences est remplie d'exemples qui illustrent éloquemment les reproches auxquels s'exposent les chercheurs qui recourent à des autorités extérieures pour faire reconnaître leurs travaux. L'épopée de la fusion froide en est un exemple récent. 
Même s'il entretient des liens directs avec les champs économique et politique, le champ scientifique dispose d'une autonomie relative qui permet à ses agents de réinterpréter et redéfinir, en fonction des intérêts et des règles propres à ce champ, les demandes et les pressions extérieures ${ }^{6}$. Toutefois, le degré d'autonomie varie, entre autres, selon les disciplines et les spécialités en fonction du volume de ressources scientifiques accumulées ${ }^{7}$. De plus, cette autonomie $n^{\prime}$ est pas parfaite dans la mesure où la mise en œuvre, le fonctionnement, l'évaluation et la consécration d'une activité scientifique ne sont jamais fondés exclusivement sur le jugement des pairs ${ }^{8}$. L'autonomie d'un groupe de scientifiques (qu'il s'agisse de membres d'une spécialité ou d'une discipline, de chercheurs universitaires ou de chercheurs de l'industrie) est donc doublement relative : elle est fonction, d'abord, de la position que le groupe occupe au sein des hiérarchies constitutives du champ scientifique et, ensuite, des relations qu'il entretient avec les champs politique et économique pour assurer son financement ${ }^{9}$. Partant de cela, on peut dire avec Michael Pollack que "la politique scientifique peut être interprétée (...) comme le lieu institutionnel des marchandages de ce degré $d^{\prime}$ autonomie relative ${ }^{10}$ ".

B Bourdieu, P. (1991), "The Peculiar History of Scientific Reason», in Sociological Forum, vol., 6, no 1, p. 6.

Bourdieu, P. (1991), op. cit., p. 15.

8 Pollack, M. (1975), «L'efficacité par l'ambiguïté. La transformation du champ scientifique par la politique scientifique : le cas de la sociologie et des sciences économiques en France", in Sociologie et sociétés, vol. 7, no 1, p. 37. 
Notre analyse s'appuie également sur la typologie qu'utilisent Ben R. Martin et John Irvine, dans Research Foresight ${ }^{11}$, en ce qui concerne les exercices de prospective de la recherche. Cette typologie nous fournit une liste détaillée des dimensions à partir desquelles les exercices de choix de priorités peuvent être étudiés. Nous en présentons maintenant les éléments les plus pertinents pour notre analyse.

\section{Les caractéristiques de l'organisme responsable de l'exercice}

Les exercices de choix de priorités relèvent de plusieurs organismes différents. II peut s'agir d'organismes publics nationaux ayant un rôle essentiellement consultatif : le Conseil des sciences du Canada, le Conseil de la science et de la technologie du Québec, etc. Toujours dans la sphère publique, l'organisme en charge de l'exercice peut aussi être responsable de programmes de soutien à l'activité scientifique et technique (par ex. : le ministère de I'Industrie, du Commerce et de la Technologie (MICT), le ministère de l'Enseignement supérieur et de la Science (MESS), le Fonds pour la formation de chercheurs et l'aide à la recherche (FCAR), le Conseil de recherche en sciences naturelles et génie, etc). L'organisme peut encore être extérieur au gouvernement : équipe de chercheurs universitaires, association industrielle ou entreprise conseil.

Évidemment, les caractéristiques de l'exercice varient en fonction de l'organisme qui en assume la responsabilitét ${ }^{12}$. Le choix de priorités sectorielles nationales par le MICT ou le MESS n'est pas un exercice identique à l'identification de domaines prioritaires pour le programme de subventions stratégiques d'un organisme, tel que le Fonds FCAR, dont le rôle est de soutenir la recherche universitaire. Dans le cas qui nous occupe, ce sont principalement les organismes consultatifs en matière de politique scientifique et technologique ainsi que les ministères voués

11 Martin, B. R. et J. Irvine (1989), Research Foresight, Priority-Setting in Science, Londres, Pinter Publishers, chapitre 2, "The nature and organization of research foresight", p. 19-42. On peut trouver une synthèse des éléments de cette typologie au tableau 2.1 (page 28) de cet ouvrage. 
directement au soutien de l'activité scientifique et technique qui retiendront notre attention.

\section{L'espace du système scientifique et technique}

Les exercices de choix de priorités se distinguent par leur degré de spécificitét ${ }^{13}$. Ils peuvent être de nature holistique, c'est-à-dire couvrir l'ensemble des disciplines et domaines scientifiques et techniques, ce qui est le cas le plus souvent des exercices nationaux visant à préciser les disciplines et les domaines que le gouvernement entend soutenir en priorité. Les exercices peuvent se situer au niveau macro et porter sur un nombre plus restreint de disciplines ou de domaines. II s'agit alors d'identifier parmi un ensemble de disciplines plus ou moins voisines (par exemple, les sciences naturelles) quelques disciplines ou domaines interdisciplinaires sur lesquels devraient être concentrées les ressources. Au niveau méso, les exercices se concentrent sur un seul domaine scientifique ou technique à l'intérieur duquel on détermine les créneaux les plus prometteurs au plan intellectuel ou socio-économique. Finalement, en ramenant les exercices à un niveau micro, on cherche à établir les développements possibles ou probables d'une seule spécialité. Nous discuterons surtout des exercices holistiques tout en abordant aussi les exercices des niveaux macro et méso.

\section{Les objectifs de l'exercice}

L'objectif le plus général des exercices de choix de priorités est sans doute de permettre une mobilisation plus efficace de l'activité scientifique et technique et, par là même, de maximiser ses retombées. De ce point de vue, l'une des caractéristiques centrales de ces exercices est le choix du type de retombées que l'on privilégie. Veut-on maximiser les retombées scientifiques, techniques, sociales, industrielles ou économiques? Quelle pondération les responsables d'un exercice font-ils de ces différents critères d'évaluation de l'activité scientifique et technique? L'examen des réponses qui ont été apportées à ces deux questions dans chaque exercice et l'étude de leur effet sur les 
orientations retenues seront un des thèmes centraux de notre analyse.

À côté de ces grands objectifs, Martin et Irvine relèvent les six fonctions intermédiaires de ce genre d'exercice $: 1$ ) énoncer les orientations générales qui devraient guider l'activité scientifique et technique nationale; 2) identifier et choisir dans l'ensemble des secteurs scientifiques et techniques, les disciplines et les domaines qui seront prioritaires pour les organismes de soutien; 3 ) rassembler et diffuser de l'information sur les développements possibles et probables des sciences et des techniques; 4) créer un consensus sur les priorités nationales en matière de science et de technologie; 5) faire la promotion de politiques gouvernementales auprès des intervenants du système de la R-D; 6) favoriser les échanges entre ces derniers ${ }^{14}$.

La gestion des tensions inhérentes aux exercices de choix de priorités

Toutes les tensions que rapportent Martin et Irvine ${ }^{15}$ concernent directement ou indirectement la composition des comités ou des équipes de travail chargés d'identifier et de choisir des domaines prioritaires. Ces tensions et les solutions qui leur sont apportées sont donc probablement les meilleurs indicateurs de la manière dont se déroule, dans le cadre de l'élaboration et de la mise en œuvre de la politique scientifique et technologique, le marchandage du degré d'autonomie du champ scientifique. Sur ce plan, la présence ou l'absence de tel ou tel groupe (les universitaires, les industriels, les représentants gouvernementaux) et leur capacité respective de prendre des décisions ou de les influencer déterminent de façon importante les orientations qui sont finalement retenues. Ces tensions sont tout simplement la forme concrète des problèmes que soulèvent la rencontre et l'affrontement, au sein du champ de la politique scientifique, d'intérêts et de formes de capital différents et divergents. Pour cette raison, l'examen des tensions relevées par Martin et Irvine occupera une place centrale dans notre analyse.

14 Martin, B. R. et J. Irvine (1989), op. cit., p. 22-24. 
La première tension vient de l'accent qui est mis sur les perspectives de la "poussée scientifique et technologique" et de la "traction du marché" lorsque vient le temps d'évaluer une discipline ou un domaine et de lui accorder (ou non) un statut de secteur prioritaire. Les potentialités des divers domaines seront-elles évaluées et hiérarchisées en fonction de leur valeur scientifique ou de l'intérêt qu'elles représentent pour la mise au point de nouveaux produits et procédés ainsi que pour le développement économique en général? Dans cette perspective, le comité qui fera l'évaluation et prendra les décisions sera-t-il composé de chercheurs ou d'industriels?

La deuxième tension renvoie à la nature de la stratégie adoptée : stratégie descendante (utop-down») ou ascendante («bottom-up»). Les comités qui décident des priorités sont-ils composés exclusivement de représentants gouvernementaux ou font-ils plutôt une large place aux producteurs et utilisateurs des connaissances scientifiques et des techniques?

La troisième et dernière tension a trait au choix du ou des acteurs à qui sont confiées la responsabilité et l'organisation de l'exercice. Ainsi, la conduite de l'exercice peut être placée sous la responsabilité de personnes ou de groupes non directement concernés ou affectés par le débat et par les résultats obtenus. On pense ici à une entreprise conseil, un groupe universitaire spécialisé en prospective, un organisme consultatif indépendant. Par ailleurs, l'exercice peut être placé sous la responsabilité d'un ou de plusieurs intervenants du système de la science et de la technologie: la communauté scientifique, l'industrie, le gouvernement.

\section{Le Groupe de prospective technologique}

Au Québec, la volonté gouvernementale d'établir des priorités sectorielles en matière de science et de technologie $n$ 'est pas très récente; elle prend forme à la fin des années 1970 et s'accentue tout au long des années 1980. Auparavant, dans les années 1960 et au début des années 1970, les ressources étaient canalisées vers la mise sur pied et le développement $d$ 'une infrastructure de la recherche scientifique : création du ministère de l'Éducation et grand effort de scolarisation; croissance accélérée du réseau universitaire; développement et diversification des disciplines et de leurs spécialités; création de centres de recherche et d'organismes subventionnaires; promotion de la formation des chercheurs et amélioration de leur compétitivité. 
Au printemps 1976, l'Office de planification et de développement du Québec (OPDQ) confie au Groupe interuniversitaire de prospective québécoise (GIPQ) ${ }^{16}$ le mandat de constituer une vaste prospective de la société québécoise axée sur 1995. Le gouvernement de l'époque comptait sur cette étude pour obtenir un portrait de l'état et du devenir de la société québécoise. Concrètement toutefois, ce travail de prospective n'était pas directement lié à un exercice de planification ou à une importante mise à jour des politiques gouvernementales.

La première étape de ce vaste projet comprenait l'analyse des sous-systèmes écologique, économique, urbain et régional, technologique et même du sous-système des valeurs. Confiée au Groupe de prospective technologique de l'École Polytechnique, l'étude du sous-système technologique avait pour objectif de fournir aux décideurs des informations et des analyses sur : 1) les éléments structurants du sous-système technologique; 2) les déséquilibres et les tensions qui l'affectent; 3) l'écologie de l'innovation; 4) la structure industrielle du Québec; 5) les dimensions technologiques des scénarios de développement envisagés pour l'avenir ${ }^{17}$.

Réalisée par des spécialistes en prospective (économistes, politicologues, ingénieurs et scientifiques), l'analyse s'appuie sur les outils méthodologiques propres à ce secteur de recherche: prévision par modèle, analyse systémique, matrices d'impacts croisés. Elle prend en compte aussi bien les développements scientifiques et technologiques que les facteurs socio-économiques. Dans l'ensemble, la qualité des données et des outils méthodologiques utilisés donnent de la crédibilité aux analyses. En fait, les secteurs identifiés ne relèvent pas uniquement de l'appréciation des chercheurs eux-mêmes.

Terminé au printemps 1977, le rapport-synthèse est une des premières tentatives de description du système québécois de la science et de la technologie. II est frappant de relire, treize ans

${ }^{16}$ Le GIPO était constitué de GAMMA (Université de Montréal et Université McGill), de l'INRS-Urbanisation, de l'École Polytechnique de Montréal, de l'École des hautes études commerciales et de I'UOAM.

17 Groupe de prospective technologique de l'École Polytechnique de Montréal (1977), Prospective socio-economique du Québec, $1^{\text {ro }}$ étape, Sous-systeme technologique (5.1), Rapport-synthese, OPDO. 
après, la présentation des "tendances lourdes". Si, parmi celles-ci, on retrouve évidemment les technologies de l'information et de l'énergie, on y remarque également le maintien de la qualité de l'environnement et, surtout, le secteur des matériaux, domaine qui n'avait pas à l'époque le rôle "générique" et central que lui accordent maintenant les spécialistes du développement scientifique et technologique. Le chapitre sur les «faits porteurs d'avenir" traite de sujets tels que la personnalisation du travail et les ateliers flexibles. Pour les auteurs du rapport, les «tendances lourdes" et les "faits porteurs d'avenir" étaient les éléments qui, en science et technologie, devaient marquer le plus profondément les décennies 1980 et 1990 . C'est donc principalement dans ces domaines que devait être concentrée l'intervention gouvernementale.

Malgré l'intérêt des résultats, ce travail de pionnier n'aura pas d'impact direct sur les décisions gouvernementales. Dans l'approche retenue, les secteurs prioritaires ont été identifiés par les prospectivistes : ni les chercheurs, ni les décideurs des milieux universitaire, industriel et gouvernemental $n$ 'ont été consultés. Cette absence de consultations directes auprès des principaux intervenants du système de la R-D et de l'innovation a fait que les résultats de l'étude du Groupe de prospective n'ont pas fait consensus. L'étude perd alors une large part de sa légitimité, premièrement, aux yeux des scientifiques puisqu'ils n'ont pas été consultés sur les tendances et sur l'intérêt des différentes options envisagées; ensuite, aux yeux des responsables gouvernementaux parce qu'ils n'ont pu faire valoir, dans l'évaluation et le choix de ces options, les objectifs généraux que leur gouvernement entend assigner aux activités scientifiques et technologiques.

En outre, aucun mécanisme précis de mise en œuvre des recommandations n'a été prévu. Le Groupe avait un statut de conseiller, et l'objectif principal de son rapport était de dégager des principes généraux devant guider les décideurs dans l'élaboration d'une politique technologique. Sur ce point, les travaux du Groupe ont donc manqué d'autorité puisque les prospectivistes $n$ 'avaient pas de liens directs avec les personnes qui, au gouvernement, étaient responsables de l'élaboration et de la mise en œuvre des politiques scientifiques et technologiques. La décision quant aux secteurs à privilégier revenait donc entièrement au gouvernement et, en l'absence d'un consensus entre les principaux intervenants, les recommandations de l'étude avaient très peu de chances d'être prises en considération. De plus, comme nous l'avons déjà mentionné, les travaux du Groupe ne s'inscrivaient pas dans un 
processus explicite d'élaboration de politique. En somme, les orientations proposées, comme bien des suggestions, pouvaient être facilement mises de côté.

Dans ce contexte, les travaux du Groupe de prospective technologique $n$ 'ont pas eu d'influence directe sur le choix des domaines scientifiques et technologiques à privilégier. À l'époque, ces choix étaient ceux des scientifiques eux-mêmes et aucune intervention gouvernementale n'est venue réduire l'autonomie relative dont ils disposaient. Jusqu'à cette date, en effet, les principales interventions du gouvernement du Québec en matière de science et de technologie (création de I'Institut de recherche d'Hydro-Québec en 1967, de l'Université du Québec en 1968, du Centre de recherche industrielle du Québec en 1969 et du Fonds FCAC en 1969) ont consisté à mettre sur pied des institutions de recherche et d'enseignement qui procurent aux scientifiques de tous les milieux les moyens financiers et matériels dont ils ont besoin pour faire de la science ${ }^{18}$. Même si, à compter des années 1970, le gouvernement provincial devient le premier responsable du développement scientifique du Québec ${ }^{19}$, jamais il n'est question qu'il intervienne dans le choix des domaines ou des questions sur lesquels porte l'effort des chercheurs. À ce niveau, la décision revient entièrement aux seuls scientifiques.

Malgré cela, les travaux du Groupe de prospective technologique constituent un moment important dans l'histoire de la politique scientifique québécoise puisque, pour la première fois, un groupe autre que les scientifiques directement concernés, c'est-à-dire les spécialistes ou les représentants de la discipline, s'est prononcé sur les orientations thématiques de l'activité scientifique.

La politique québécoise de la recherche scientifique : Un projet collectif

À peine quelques mois après la parution du rapport du Groupe de prospective technologique, la scène de la politique

18 Pour une histoire détaillée du processus qui mène à la création de ces institutions, voir Chartrand, L., Duchesne, R. et Y. Gingras (1987), Histoire des sciences au Québec, Montréal, Boréal, chapitre 9. 
scientifique allait changer radicalement avec l'arrivée au pouvoir du Parti québécois. Comme le soulignent Chartrand, Duchesne et Gingras, "le nouveau gouvernement [souverainiste] associe résolument la science et la technologie au projet de société qu'il propose aux Québécois; au moins par le pouvoir du discours politique, le développement scientifique se trouve étroitement intégré au développement social et culturel du Québec ${ }^{20}$ ".

Dès son entrée en fonction, le gouvernement entreprend une importante réflexion sur sa politique scientifique. C'est dans le Livre blanc sur la politique culturelle, paru en 1978, que sont présentées les premières indications des orientations qu'il entend privilégier $^{21}$. La science y est considérée sous deux angles: d'abord comme bien culturel, puis comme force de production ${ }^{22}$. Déjà dans cet énoncé de politique, le gouvernement indique son intention de mettre en œuvre une politique scientifique qui permettra de choisir les domaines scientifiques et techniques à privilégier et d'arrimer ces choix avec les priorités sociales, culturelles et économiques du Québec ${ }^{23}$.

Dans l'ensemble, on retrouve les mêmes orientations dans le Livre vert sur la politique scientifique que le gouvernement publie en mars 1979 et qui fait le point sur quinze ans d'efforts et de réformes dans le domaine de la $R-D^{24}$. L'analyse de l'activité

20

Chartrand, L., Duchesne, R. et Y. Gingras (1987), op. cit., p. 303.

21 Gouvernement du Québec, ministre d'État au développement culturel (1978), La politique québécoise du développement culturel. Les trois dimensions d'une politique : genres de vie, création, éducation, Québec, Éditeur officiel, 2 vol.

22 Gouvernement du Québec, ministre d'État au développement culturel (1978), op. cit., p. 276.

23 Gouvernement du Québec, ministre d'État au développement culturel (1978), op. cit., p. 277-278.

24 Gouvernement du Québec, ministre d'État au développement culturel (1979), Pour une politique québécoise de la recherche scientifique, Québec, Éditeur officiel. 
scientifique est cependant beaucoup plus poussée que dans $\mathrm{La}$ politique québécoise du développement culturel, et le gouvernement indique clairement son intention de se doter des outils, entre autres d'une structure gouvernementale, qui lui permettront de faire une véritable planification de la recherche scientifique et technique ${ }^{25}$.

Un des principaux thèmes du Livre vert est la question des priorités sectorielles. $\mathrm{Si}$, à cet égard, il n'est jamais fait mention des secteurs et des domaines scientifiques et techniques dans lesquels devraient être concentrées les ressources, le gouvernement insiste cependant sur la nécessité de faire des choix et de développer les conditions et les mécanismes permettant de telles prises de décision. Refusant de procéder unilatéralement au choix des priorités, il invite plutôt les chercheurs et les institutions qui les représentent à participer à l'élaboration «des orientations et des objectifs prioritaires à poursuivre, et même des modalités d'organisation à mettre sur pied ${ }^{26}$ ".

Lors de la vaste consultation publique qui a suivi la publication du Livre vert, les chercheurs et leurs associations sont de loin les plus actifs. Un plus grand nombre de mémoires provenait des milieux universitaires que des représentants des milieux industriels ou gouvernementaux.

Sur l'opportunité de favoriser le développement de la R-D dans quelques domaines prioritaires correspondant aux besoins et objectifs de la société québécoise, les opinions des différents intervenants vont de l'accord au rejet complet. Les opposants, principalement les universités, mais aussi quelques représentants des milieux industriels, soulignent leur crainte de voir l'État imposer de trop grandes contraintes politiques et bureaucratiques qui mettraient en danger la liberté du chercheur et sa capacité de développer des initiatives créatrices. À l'opposé, ceux qui appuient l'orientation proposée dans le Livre vert affirment que le Québec

25 Gouvernement du Québec, ministre d'État au développement culturel (1979), op. cit., p. 178.

26 Laurin, Camille, «Allocution du Docteur Camille Laurin, ministre d'État au développement culturel, prononcée lors du lancement du Livre vert", in Gouvernement du Québec, ministre d'État au développement culturel (1980a), Pour une politique québécoise de la recherche scientifique. La consultation, Québec, Éditeur officiel, p. 8. 
n'aura jamais à sa disposition les moyens financiers nécessaires à la poursuite de travaux dans tous les secteurs de R-D et ils insistent sur la pertinence de développer des mécanismes permettant de faire des choix conscients et concertés. Sur les mécanismes à mettre au point, tous les intervenants semblent s'entendre : si les ressources consacrées à la R-D doivent être concentrées dans des secteurs prioritaires, les mécanismes conduisant à leur identification devraient permettre une participation importante et déterminante des chercheurs ${ }^{27}$. Comme on peut facilement le constater, les participants à la consultation négociaient le degré d'autonomie du champ scientifique : certains, principalement l'État, cherchaient à la réduire tandis que $d$ 'autres, surtout les chercheurs universitaires, essayaient de conserver une grande autonomie en continuant d'avoir une place privilégiée dans les mécanismes décisionnels.

Cette consultation a été suivie d'un Livre blanc, Un projet collectif, qui pave la voie aux actions des années 1980 . Tout en réservant une place à la recherche libre, le document traite de la nécessité de déterminer démocratiquement les priorités de la recherche.

Articulée à l'ensemble des politiques de l'État québécois, la politique scientifique va dorénavant avoir pour objectif de veiller à ce que l'activité scientifique et technique contribue activement au développement culturel et social de la société québécoise ainsi qu'à sa croissance économique et à l'amélioration de la qualité de vie de sa population. Partant de là, le gouvernement indique que la science ne peut plus être laissée aux mains des seuls chercheurs et que la communauté scientifique devra tenir compte des priorités collectives établies dans l'ensemble des politiques de développement du gouvernement.

Cherchant à instituer un dialogue entre les représentants de l'État et la communauté scientifique, le gouvernement propose un nouveau mécanisme de détermination des priorités :

L'État ne se livrerait donc généralement pas à des énumérations de priorités de recherche proprement dites, mais donnerait plutót son appui à des projets

27 Gouvernement du Québec, ministre d'État au développement culturel (1980a), op. cit. 


\begin{abstract}
scientifiques élaborés par la
communauté scientifique et publiquement discutés dans les différentes instances consultatives et décisionnelles du système scientifique : la pertinence de ces projets par rapport aux priorités collectives de développement déjà énoncées serait alors le principal critère auquel l'État se référerait pour les promouvoir et pour les appuyer financièrement ${ }^{28}$.
\end{abstract}

Le gouvernement entend ainsi tenir compte des besoins des utilisateurs (entreprises, gouvernements et citoyens) dans la détermination des priorités sectorielles en recherche scientifique et technique.

À cet égard, il est intéressant de remarquer que les représentants des milieux industriels ont peu participé aux discussions. Déjà faiblement représentés lors des consultations tenues à la suite du Livre vert, les industriels et dirigeants d'entreprise sont absents des mécanismes de détermination des priorités proposés dans le Livre blanc. Absence qu'explique au moins en partie l'objectif du Livre blanc qui était de définir une politique de la recherche scientifique et non une politique de développement technologique. Néanmoins, compte tenu du lien que le gouvernement désirait établir entre sa politique de la recherche scientifique et le développement économique du Québec, cette absence a quelque chose d'étonnant.

Sans la participation réelle des industriels, ce sont les facteurs de la demande qu'a négligés le gouvernement. Plutôt que de laisser les représentants des entreprises exposer leurs orientations et exprimer leurs besoins, le gouvernement a procédé à l'identification et au choix des priorités en collaboration seulement avec les chercheurs. En regard des objectifs de développement économique et social assignés aux activités de recherche, l'absence de représentants du monde industriel constitue un problème majeur.

${ }^{28}$ Gouvernement du Québec, ministre d'État au développement culturel (1980b), Un projet collectif. Enoncé d'orientations et plan d'action pour la mise en œuvre d'une politique québécoise de la recherche scientifique, Québec, Éditeur officiel, p. 19. 
Comme le soulignent Chartrand, Duchesne et Gingras, tant le Livre vert de 1979 que le Livre blanc de 1980 ont été I'occasion pour le gouvernement de revendiquer «aux dépens d'Ottawa ou du secteur privé la responsabilité première de la recherche scientifique et technique au Québec ${ }^{29}$ ". Dorénavant, l'État québécois sera un intervenant important dans la planification de l'activité scientifique et il entend jouer un rôle actif dans le choix des domaines auxquels seront consacrées les ressources. Mieux traités que les représentants du monde industriel, les chercheurs universitaires ne sont pas totalement exclus des mécanismes de prise de décision. Cependant, le gouvernement indique clairement qu'ils ne seront plus les seuls à décider et qu'ils devront composer avec les organismes gouvernementaux chargés de définir plus précisement et de mettre en œuvre la nouvelle politique scientifique. En somme, on constate qu'au terme de ce long processus de réflexion sur la politique scientifique, les chercheurs, qu'ils soient de l'industrie ou des universités, ont perdu une part de l'autonomie dont ils avaient disposé jusque-là . Les instances décisionnelles de l'État en matière de science et de technologie utiliseront comme principal critère d'évaluation la pertinence d'un projet par rapport aux priorités collectives de développement énoncées par le gouvernement. Dans ces nouvelles orientations, il n'est même plus question du mérite et de l'intérêt scientifiques du projet!

\section{Le Conseil de la politique scientifique}

Les nouveaux organismes créés dans la foulée du Livre blanc vont $s^{\prime}$ attaquer très rapidement au mandat de planification de l'activité scientifique et technique que leur a confié le gouvernement. Dès l'automne 1981, le Conseil de la politique scientifique du Québec (CPSQ) décide de lancer une opération d'identification des domaines prioritaires en recherche et développement. Le but de cette opération: faire des recommandations au ministre responsable de la politique scientifique $^{30}$. Pour le CPSQ, une petite collectivité comme le Québec ne peut se permettre de couvrir l'ensemble des activités

29 Chartrand, L., Duchesne, R. et Y. Gingras (1987), op. cit., p. 304.

30 Conseil de la politique scientifique du Québec (1983), Les priorités du domaine scientifique et technique, Rapport interne, 83-03-08, p. 2. 
scientifiques et techniques. Si elle désire maximiser l'impact de ces activités sur son développement économique et socioculturel, elle ne doit pas répartir ses ressources de manière quasi uniforme sur l'ensemble des activités possibles. Comme cela avait été souligné dans le Livre blanc sur la recherche scientifique, le CPSO estime qu'il faut faire des choix, c'est-à-dire reconnaître et déterminer des domaines prioritaires qui soient adaptés aux objectifs économiques et sociaux de la société québécoise.

Dans sa problématique, le CPSO distingue l'identification et le choix des priorités. L'identification relève essentiellement de la consultation de tous les intervenants concernés par les activités de l'organisme qui procède à l'exercice. En accordant une place aussi importante à la consultation, la démarche proposée par le CPSO suit l'une des orientations du Livre blanc. Toutefois, à la différence de ce qui était prévu dans ce document, il élargit la participation et se propose de convier les représentants des milieux industriels à participer. Par ailleurs, le choix des secteurs et la décision relative aux ressources devant être consacrées à chaque priorité relève exclusivement des décideurs gouvernementaux. Là aussi il respecte l'esprit et les orientations du Livre blanc.

Le CPSO entend délimiter clairement la portée de son travail et décide de centrer son opération sur les domaines de l'activité scientifique ayant une composante scientifique élevée et un fort potentiel de développement économique. L'objectif de la démarche est clair, et les participants disposent de critères leur permettant de mieux évaluer et comparer les différents secteurs de R-D. Point intéressant: le CPSO centre son exercice sur le potentiel économique des activités scientifiques et met donc de côté les objectifs de développement social et culturel qui étaient si importants dans le Livre blanc.

La démarche s'amorce à la fin de 1981 par une enquête Delphi menée auprès de membres du Conseil provenant des universités, du gouvernement provincial et des entreprises. L'enquête permet de relever dix-sept secteurs :

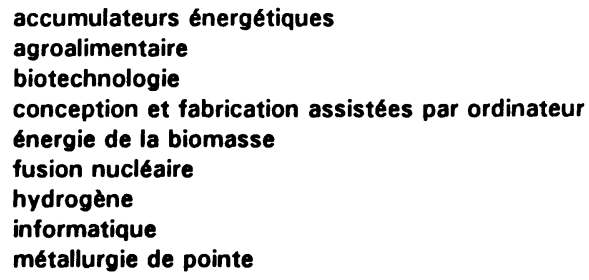


- méthodes de construction

- microélectronique

- $\quad$ produits pharmaceutiques

- robotique

- technologies électrotechniques

- télématique

- utilisation des résidus du bois

- véhicules électriques ${ }^{31}$.

Le Conseil demande ensuite à des spécialistes de préparer une analyse préliminaire de chacun de ces secteurs en utilisant une série de critères dont les trois principaux sont : le niveau des ressources humaines et financières requis pour assurer le développement du secteur; l'ampleur de l'impact et des retombées positives et négatives associés à ce développement; et les avantages comparatifs du Québec sur la scène internationale.

Sur la base de ces travaux, le Conseil entreprend de regrouper les secteurs par affinités et met sur pied trois comités d'experts qui, utilisant les mêmes critères que lors des analyses préliminaires, font l'analyse des trois domaines suivants : informatique, biosciences, énergie et transports. Les experts ayant participé à la rédaction des analyses préliminaires centrées sur les secteurs d'un même domaine sont invités à siéger au comité responsable.

Malheureusement, les travaux du CPSO s'arrêtent à cette étape. La Loi 19 remet tout en question, à commencer par le Conseil lui-même qui devient, peu après, le Conseil de la science et de la technologie. Au moment où cessent les travaux du CPSO, deux des trois comités sont sur le point de terminer leur rapport et le Conseil de la science et de la technologie décide donc de parachever le travail. L'étude sur l'informatique est publiée par le Conseil au début de 1984 et celle sur les biosciences, rebaptisées biotechnologies, paraît en 1985 . Quant à celle sur l'énergie et les transports, elle ne sera jamais terminée.

Les efforts du Conseil de la science et de la technologie permettent de rendre public et de récupérer, au moins en partie, le résultat du travail des comités. Toutefois, le contenu des deux études publiées reste bien en deçà des ambitions énoncées par le CPSO, entre autres parce que l'exercice a été interrompu avant que les trois comités d'experts $n^{\prime}$ aient eu le temps de soumettre leurs rapports à une large discussion publique et parce que, ces

31 Conseil de la politique scientifique du Québec (1983), op. cit., Annexe. 
consultations n'ayant pas eu lieu, il devenait difficile d'établir un consensus autour des secteurs à privilégier.

Comparée aux tentatives antérieures, la démarche du CPSO présente néanmoins des caractéristiques distinctives qui révèlent certains traits importants de la politique scientifique de l'époque. Elle repose sur la consultation de tous les intervenants; elle vise un objectif bien défini; et l'évaluation des différents secteurs de R-D s'appuie sur une série de critères précis et bien adaptés à l'objectif général de la démarche. Malgré cela, les travaux du CPSO n'ont pas eu d'impact direct et immédiat sur les décisions gouvernementales. Une des raisons en est que la démarche du CPSQ a été une initiative interne: elle ne répondait ni à une demande explicite des ministres concernés par le financement de la $R-D$, ni à un besoin exprimé par les autres intervenants du sytème de la science et de la technologie. L'étude du CPSO n'a donc pas eu de "client». Sur un autre plan, la démarche proposée par le CPSQ fait aussi problème parce qu'elle ne prévoit aucun mécanisme de mise en œuvre des priorités suggérées. Comme le fait l'étude du Groupe de l'École Polytechnique, elle sépare la reconnaissance du choix des priorités et n'intègre donc pas les représentants gouvernementaux qui pourraient influencer directement la formulation des politiques et les processus d'allocation des ressources. Et au moment où le CPSO poursuit ses travaux, rien $n$ 'indique que les ministres responsables $d u$ dossier science et technologie soient prêts à choisir des domaines prioritaires pour l'activité scientifique et technique.

L'historique de l'étude du CPSO montre que la volonté du gouvernement d'intervenir dans le choix de domaines prioritaires, présente dans le Livre blanc, se matérialise avec difficulté. On constate que, $d$ 'une part, les ministres responsables des questions scientifiques et technologiques n'ont pas eu l'initiative de l'exercice du Conseil et que, d'autre part, ils n'y ont même pas été associés directement. Ainsi, à cette époque, le gouvernement n'a procédé au choix de secteurs prioritaires pour la R-D ni en se basant sur l'étude du CPSQ, ni en s'appuyant sur d'autres études. En somme, on peut dire que si le discours politique du Livre blanc et la création subséquente d'organismes responsables de la politique scientifique menaçaient l'autonomie du champ scientifique, les actions qui ont suivi ont laissé cette autonomie largement intacte. 
Le virage technologique

Avant même que toutes les mesures envisagées dans le Livre blanc n'aient été mises en œuvre, la crise économique de 1982 obligeait le gouvernement à réviser ses orientations en matière de science et de technologie. Le lien entre science et culture, qui avait été mentionné dans tous les énoncés de politique scientifique du gouvernement depuis 1978, est mis de côté au profit de la relation entre science-technologie et développement économique. Dans cette perspective, l'activité scientifique et technique est mise au service d'une restructuration en profondeur de l'économie québécoise. Combinée à un contexte d'urgence, cette définition plus restrictive de la direction que doit prendre l'activité scientifique et technique va permettre au discours interventionniste des années précédentes de se matérialiser.

C'est dans Le virage technologique, seconde phase d'un programme d'action économique, que l'on retrouve les nouvelles orientations du gouvernement. Dans le contexte d'une libéralisation croissante des échanges, le Québec, déclare le gouvernement, doit assurer la croissance de ses entreprises, profiter des grands projets, développer sa compétence, miser sur la microélectronique et les biotechnologies ainsi que sur ses atouts naturels (énergie, agroalimentaire, pêches maritimes, forêts, mines, transports et tourisme). Pour le gouvernement, ces secteurs constituent autant de domaines de R-D où il devrait concentrer ses efforts afin d'accélérer et de renforcer le développement économique ${ }^{32}$.

Le virage technologique propose aussi une nouvelle vision de la croissance économique en affirmant qu'elle est de plus en plus liée à la perception juste des situations et à la capacité de réagir rapidement et avec souplesse aux changements. Dans cette nouvelle vision, la veille scientifique et technologique peut, potentiellement, jouer un rôle important. De plus, la concertation est vue comme un moyen de susciter chez l'ensemble des

32 Gouvernement du Québec, Développement économique (1982), Le virage technologique, Bâtir le Québec - Phase 2, Programme d'action économique 1982-1986, Québec, ministère des Communications. 
partenaires un engagement de nature à favoriser la réalisation des objectifs prioritaires définis par consensus ${ }^{33}$.

En 1984, lors de l'appel de propositions pour le Programme d'actions structurantes pour le soutien d'équipes de recherche, la Direction de l'enseignement et de la recherche universitaires (ministère de l'Éducation) a utilisé la liste de secteurs prioritaires identifiés dans $L e$ virage et limité le concours à sept domaines de recherche :

- la biotechnologie
l'informatique
- les nouvelles technologies électroniques
l'énergie
- le transport
l'agroalimentaire
la maîtrise sociale des changements technologiques.

Le Programme d'actions structurantes met l'accent sur la coordination des efforts de recherche, la complémentarité des composantes du système universitaire et la formation du personnel scientifique. Ce faisant, il vise à assurer le développement, la consolidation et, si nécessaire, l'émergence d'unités de recherche. Le gouvernement espère que les 50 millions de dollars qu'il entend consacrer à ce programme permettront de créer des pôles d'excellence dans des secteurs stratégiques, ce qui favorisera le développement économique de la province.

L'arrivée au pouvoir du Parti libéral en décembre 1985 ne modifiera pas radicalement les grandes orientations gouvernementales en matière de R-D. Ainsi, en juin 1987, le ministre de l'Industrie et du Commerce, Daniel Johnson, réaffirmait la volonté du gouvernement de lier politique industrielle et politique scientifique et technologique. II parlait, entre autres, de la nécessité d'identifier des secteurs d'activités où les investissements doivent être attirés et encouragés, pour donner lieu à la croissance de l'emploi ${ }^{34}$.

33 Gouvernement du Québec, Développement économique (1982), op. cit., Introduction générale, p. 13-26.

34 Johnson, D. (1987), «Notes d'allocution de Daniel Johnson, ministre de I'Industrie et du Commerce, a l'occasion de l'ouverture des travaux de la Commission de l'économie et du travail portant sur l'étude des crédits 1987-1988 du ministère de l'Industrie et du Commerce», Assemblée nationale 
Pour le ministre, ces secteurs prioritaires sont ceux où le Québec dispose d'avantages comparatifs naturels et possède déjà une expertise scientifique et technique. Ils sont aussi ceux qui entraînent les retombées économiques les plus importantes et qui recèlent un fort potentiel de restructuration du tissu industriel québécois. La combinaison de ces critères donne au ministre une liste qui recoupe, en majeure partie, celle proposée dans $L e$ virage technologique et reprise dans le Programme d'actions structurantes :

- les industries énergivores

- les industries des véhicules et des pieces

- l'aérospatiale et les produits de la défense

- l'industrie pharmaceutique et la biotechnologie du secteur de la santé

- la microélectronique, les télécommunications, l'informatique

- les produits en matière plastique ${ }^{35}$.

Dans les trois situations que nous venons de présenter, $c^{\prime}$ est le gouvernement qui, isolément, a identifié et choisi les secteurs prioritaires au plan économique et, par là même, les secteurs de R-D devant être financés prioritairement compte tenu des liens étroits qu'il veut établir entre les activités de R-D et le développement économique. Depuis Le virage technologique, l'État québécois se montre plus interventionniste qu'auparavant et, en dépit de la très grande importance qu'il accorde à la concertation, il travaille seul, négligeant ainsi le point de vue et les opinions des représentants de la communauté scientifique et du monde de l'industrie et des affaires.

De plus, contrairement à ce qui s'était passé au lendemain du Livre blanc sur la recherche scientifique, le gouvernement est parvenu à concrétiser ses orientations et il a ainsi décidé, au fil des ans, de créer plusieurs centres de recherche dans les secteurs qu'il avait déclaré prioritaires : entre autres, le Centre québécois de valorisation de la biomasse, le Centre québécois d'informatisation de la production, le Centre de recherche sur les applications pédagogiques de l'ordinateur, le Centre de recherche informatique de Montréal, le Centre francophone de recherche en informatisation des organisations, I'Institut de technologie du magnésium, le

du Québec, le 1er juin 1987, p. 2. 
Centre de recherche en microélectronique (Université de Sherbrooke) et l'Institut de recherche en pharmacie industrielle.

\section{Le Sommet québécois de la technologie}

À l'été 1988, le nouveau ministère du Commerce extérieur et du Développement technologique publie un document de consultation intitulé La maîtrise de notre avenir technologique : un defi à relever ${ }^{36}$. Par ce geste, le gouvernement veut montrer l'importance qu'il accorde au dossier de la science et de la technologie. Par ailleurs, il désire aussi effectuer une mise à jour de sa politique scientifique et technologique.

Cette nouvelle politique s'articule autour de deux projets. Le premier est de porter les dépenses québécoises de R-D à $2 \%$ du PIB en 1992. Pour ce faire, le gouvernement compte sur une augmentation des dépenses des entreprises et du gouvernement fédéral. De son côté, il gèle ses contributions directes et compense cette stabilisation par d'importantes mesures fiscales destinées à favoriser le développement des activités de R-D dans les entreprises. Le deuxième projet consiste en la création d'un fonds de développement technologique de $\mathbf{3 0 0}$ millions de dollars destiné à soutenir des programmes tant dans les entreprises que dans les universités ${ }^{37}$.

Cette nouvelle politique, le gouvernement veut la placer sous le signe de la concertation et du consensus. II n'identifie pas lui-même des priorités sectorielles et convie tous les intervenants du système québécois de la R-D à venir discuter des orientations qu'il propose dans le cadre du Sommet québécois de la technologie. Le gouvernement espère que le Sommet permettra de dégager les consensus sur la base desquels il pourra ensuite, en collaboration avec ses partenaires, établir son plan d'action ${ }^{38}$.

${ }^{36}$ Gouvernement du Québec, ministère du Commerce extérieur et du Développement technologique (1988), La mañtrise de notre avenir technologique : un défi à relever.

37 Conseil de la science et de la technologie (1988), Science et technologie. Conjoncture 1988, Sainte-Foy, p. 135. 
Du 12 au 14 octobre 1988, un peu plus de 400 représentants des milieux de l'industrie, de l'enseignement supérieur et de l'État se sont réunis à Montréal avec l'objectif de susciter "au sein de la société québécoise une meilleure appréciation des défis de nature technologique et compétitive qui la confrontent" et de dégager "un certain nombre d'idées directrices et de priorités qui baliseront l'action ultérieure de chacun des partenaires ${ }^{39}$ ".

Partant de la prémisse que les conditions du développement technologique varient selon les secteurs industriels, les responsables du Sommet ont décidé que les ateliers de travail seraient organisés de manière à rassembler au sein d'un groupe de discussion les entrepreneurs, les chercheurs universitaires et les responsables gouvernementaux qui sont directement ou indirectement intéressés par un même secteur industriel.

Pour l'identification de domaines prioritaires en R-D, cette façon de procéder a été très efficace. Dans plusieurs ateliers, elle a permis de relever les forces et les faiblesses du secteur et, dans la même foulée, $d$ 'indiquer les niches spécifiques dans lesquelles le Québec devrait investir compte tenu des possibilités économiques qu'elles représentent et des avantages dont le Québec dispose. Par exemple, les participants à l'atelier sur les biotechnologies ont reconnu quatre domaines d'activités dans lesquels le Québec possède des atouts intéressants: le biomédical, la foresterie, la bioénergie et l'environnement. C'était la première fois que les efforts portaient sur l'identification de créneaux plutôt que celle de secteurs qui, en règle générale, restent trop larges et trop nombreux pour permettre une véritable concentration des ressources.

Par contre, ce type d'organisation rend plus difficiles la comparaison et la hiérarchisation des secteurs. En fait, la question des secteurs prioritaires n'était pas à l'ordre du jour des séances plénières; elle $n^{\prime} y$ a été ni abordée ni débattue. De plus, l'information produite dans les ateliers n'a permis au comité organisateur de faire qu'une évaluation très sommaire de la position de chaque secteur sur la scène internationale. Cela a évidemment compliqué l'identification des domaines ou créneaux scientifiques

39 Lortie, P. (1988), «Rapport du comité organisateur», in Sommet québécois de la technologie (1988), Compte rendu des délibérations, Québec, p. 1. 
et techniques dans lesquels le Québec est le mieux placé et le plus compétitif.

Dans le compte rendu des délibérations du Sommet, on retrouve plusieurs listes de secteurs prioritaires. Le comité organisateur mentionne que le Québec dispose de forces particulières dans les domaines de la biotechnologie, des télécommunications, de l'aérospatiale et dans quelques autres secteurs qu'il ne nomme cependant pas. Dans le chapitre qui présente la synthèse thématique des principaux éléments soulevés dans les ateliers, on peut lire que les pâtes et papiers, les télécommunications, l'aéronautique et les produits pharmaceutiques sont des secteurs dans lesquels le Québec a du succès et sur lesquels doit s'appuyer son développement économique. Dans le même chapitre, on peut trouver une autre liste sous le thème des projets mobilisateurs: l'environnement, l'énergie et l'hydrogène, les technologies de transports publics, l'informatisation de la production, la télématique, la géomatique, l'aménagement forestier et la valorisation des ressources forestières $^{40}$.

Même s'ils n'ont pas travaillé à établir une liste bien définie de secteurs prioritaires, les participants ont exprimé néanmoins une préférence très marquée pour une stratégie de niches. En ce sens, plusieurs d'entre eux ont adopté une position très éloignée de celle qu'ils avaient défendue en 1980, lors de la consultation sur la politique québécoise de la recherche scientifique.

Les participants au Sommet ont souligné leur opposition aux politiques gouvernementales qui conduisent au "saupoudrage" des ressources dans un grand nombre de secteurs et sur une multitude de projets. S'inspirant de l'exemple de pays comme la Norvège et la Suède, ils ont insisté sur la nécessité de penser et de mettre en œuvre des stratégies permettant de concentrer les efforts sur des créneaux "porteurs" afin d'accélérer le développement technologique du Québec ${ }^{41}$.

Par ailleurs, et ce malgré l'importance qu'il accorde à la concertation dans son discours politique, le gouvernement n'a pas attendu les résultats du Sommet pour officialiser sa nouvelle politique. Dans le discours d'ouverture, le Premier ministre a

40 Lortie, P. (1988), op. cit., p. 6; chapitre III, p. 2 et 9.

41 Lortie, P. (1988), op. cit., p. 6 et 7. 
annoncé une injection additionnelle de 1,39 milliard de dollars en cinq ans : 760 millions sous forme de mesures fiscales, 330 millions dans le cadre d'une politique d'impartition pour les dépenses du gouvernement et d'Hydro-Québec et $\mathbf{3 0 0}$ millions libérés pour la création d'un nouveau fonds destiné au développement technologique ${ }^{42}$. Établi pour cinq ans, le Fonds de développement technologique (FDT) financera des «projets mobilisateurs" qui visent l'adoption, l'adaptation et la mise au point de nouveaux procédés ou produits commercialisables. Le Fonds cherche aussi à réunir autour d'un même projet les secteurs privé, public et universitaire.

Dans la description des objectifs visés par le FDT, rien n'indique que certains secteurs pourront être privilégiés et le bref exposé sur les projets mobilisateurs mentionne vaguement qu'il doit s'agir de projets de développement technologique d'envergure dans des domaines forts ou porteurs d'avenir pour l'économie du Québec, domaines non précisés ${ }^{43}$. De plus, rien dans les modalités d'évaluation des demandes ne prévoit que le secteur dans lequel s'inscrit un projet pourra constituer un critère de sélection.

Par ailleurs, l'absence d'une liste de secteurs prioritaires sur laquelle se serait appuyée la gestion du Fonds n'a pas empêché le gouvernement de décider seul qu'une partie des sommes disponibles serait dépensée dans des domaines ou créneaux spécifiques. Ainsi, au budget de $\mathbf{3 0 0}$ millions de dollars, prévu à l'origine, sont venus s'ajouter 50 millions réservés à la recherche sur l'environnement. De la même manière, le gouvernement a décidé d'utiliser le FDT pour financer sa contribution au projet RADARSAT (32 millions) et à l'Institut de la technologie du magnésium (2,8 millions). Finalement, près de 4,5 millions de dollars seront prélevés à même le Fonds pour payer les frais

42 Bourassa, R. (1988), «Notes pour l'allocution du Premier Ministre du Québec, Robert Bourassa, à l'occasion de l'ouverture du Sommet québécois de la technologie", in Sommet québécois de la technologie (1988), op. cit.

43 Gouvernement du Québec (1989), Imaginons demain, Le Fonds de développement technologique. Des projets mobilisateurs, p. 4 et 5. 
indirects associés à la participation des universités québécoises au Réseau fédéral de centres d'excellence ${ }^{44}$.

Dans l'ensemble, les événements qui ont marqué la politique scientifique et technologique du Québec depuis 1988 montrent clairement que la concertation dont se réclame le gouvernement est restée du domaine du discours politique. Comme à la fin des années 1970 et au début des années 1980, le gouvernement a élaboré et arrêté ses principales orientations sans vraiment tenir compte de l'avis des autres intervenants du système scientifique et technologique. À la différence des politiques antérieures, toutefois, la politique de 1988 ne comprend pas de liste de secteurs ou domaines prioritaires. Ainsi, le FDT, un des plus importants programmes de soutien à la science et à la technologie, ne sera pas l'occasion de planifier la concentration des efforts du Québec dans quelques domaines de R-D jugés prioritaires. De la même manière, les mesures fiscales adoptées par le gouvernement ne sont pas restreintes aux activités de R-D qui s'insèrent dans une liste préétablie de secteurs ou domaines prioritaires.

La communauté scientifique, et principalement les chercheurs universitaires, qui, entre 1978 et 1987, ont tellement craint que l'État $n$ 'intervienne directement et sans réserve dans le choix des domaines et des questions de recherche ont moins de raisons de s'inquiéter : ce n'est le plus souvent qu'à la marge que la nouvelle politique du gouvernement du Québec attaque l'autonomie du champ scientifique. Comme le montre l'examen des deux principales mesures de soutien, c'est-à-dire le FDT et les mesures fiscales, le soutien financier de l'État, dans sa quasi-totalité, ne repose pas inconditionnellement sur la concordance des projets de recherche avec les choix de priorités gouvernementales. Les scientifiques, qu'il s'agisse de chercheurs individuels, d'équipes universitaires ou de départements de R-D dans les entreprises, conservent donc une très large part de leur capacité à déterminer eux-mêmes les domaines de recherche sur lesquels ils veulent faire porter leurs efforts.

44 Conseil de la science et de la technologie (1991), Science et technologie. Conjoncture 1991, Sainte-Foy, p. 31. 


\section{La politique scientifique québécoise et les priorités fédérales}

Au Québec, les scientifiques sont, d'une certaine façon, confrontés à deux politiques : celle du gouvernement du Québec et celle du gouvernement fédéral. Chaque niveau de gouvernement a sa propre politique scientifique et technologique ainsi que ses propres organismes responsables de définir et de mettre en œuvre des programmes de soutien à l'activité scientifique et technique. Ce dédoublement s'applique aussi au choix de domaines prioritaires pour la R-D : chaque niveau de gouvernement établit ses propres listes de priorités sectorielles, lesquelles ne sont jamais parfaitement identiques.

Sans faire une histoire détaillée de la politique scientifique et technologique canadienne, on peut néanmoins constater que le gouvernement fédéral se soucie très peu des priorités sectorielles retenues par les gouvernements provinciaux. Qu'il s'agisse des secteurs désignés pour le Programme des technologies stratégiques (biotechnologies, informatique et matériaux industriels avancés), pour les programmes spéciaux d'Industrie, Science et Technologie (Programme de la microélectronique et du développement des systèmes, Programme d'impartition dans le domaine de l'intelligence artificielle, Fonds de la stratégie nationale en biotechnologie) ou, enfin, pour les programmes d'aide à la recherche et à l'innovation industrielles du même ministère (forêts, pêcheries, secteur manufacturier avancé, automobile, environnement, appareils médicaux), les choix sont faits par le gouvernement fédéral après identification et évaluation des besoins nationaux. $\mathrm{Si}$, à certaines occasions ${ }^{45}$, le fédéral a consulté les représentants des milieux industriels et universitaires canadiens avant d'identifier et de choisir des domaines ou créneaux prioritaires, il a toutefois laissé de côté les représentants des gouvernements provinciaux.

45 On pense, par exemple, aux travaux du Comité consultatif national du ministère d'État à la Science et à la Technologie qui, dans le but de spécifier des créneaux prioritaires pour chacune des trois technologies génériques du Programme des technologies stratégiques met en œuvre un vaste exercice de consultation auquel participent activement les représentants des universités et de l'industrie. Les recommandations issues de cette consultation seront par la suite reprises dans les orientations qui sont données à la Stratégie nationale en biotechnologie et au Programme de la microélectronique et du développement des systèmes. 
Cette indifférence aux priorités sectorielles de l' "autre" n'est pas le propre du gouvernement fédéral et, comme on l'a vu précédemment, le gouvernement du Québec s'est lui aussi fort peu soucié de celles du gouvernement canadien. Au Canada, l'intégration des priorités sectorielles des gouvernements provinciaux à celles du gouvernement fédéral a été souvent négligée par les différents intervenants. Le gouvernement fédéral, par exemple, lors du choix des secteurs et créneaux pour son Programme des technologies stratégiques, n'a pas tenu compte des secteurs prioritaires déterminés par les provinces.

Ces problèmes de cohérence créent des situations où un secteur jugé prioritaire par une province connaîtra un développement beaucoup plus lent faute d'un investissement fédéral important. Au Québec, par exemple, le développement du secteur de la fusion nucléaire, que le Conseil de la politique scientifique avait jugé prioritaire en 1983 et qui a toujours bénéficié du soutien du gouvernement du Québec, a été longtemps retardé parce qu'il n'était pas prioritaire pour le gouvernement fédéral ${ }^{46}$.

En fait, que ce soit au Canada ou dans les autres pays industrialisés, les gouvernements centraux ne tiennent généralement pas compte des forces et faiblesses des régions (ou provinces) lors du choix des priorités nationales. Par conséquent, certaines régions (ou provinces) voient des domaines où elles sont "fortes", exclus des priorités nationales. D'autres qui cherchent à développer leurs activités scientifiques et technologiques en misant sur leurs points forts, peuvent ainsi privilégier des domaines qui ne font pas partie des priorités nationales.

II ne faudrait pas toutefois exagérer ce manque de cohérence. II arrive que les deux niveaux de gouvernement privilégient les mêmes domaines: biotechnologies, microélectronique, nouveaux matériaux. Et même si le gouvernement fédéral, dans chaque technologie générique, détermine toute une série de créneaux plus spécifiques, le risque que des priorités provinciales soient exclues est minime. En fait, l'adéquation entre un projet et les créneaux reconnus prioritaires dans le cadre d'un programme ne constitue jamais un critère central d'évaluation. Par ailleurs, on peut aussi dire que, pour les

46 Pour plus de détails concernant le développement de la recherche sur la fusion nucléaire au Québec, voir Gingras, Y. et M. Trépanier (1989), «Le Tokamak de Varennes et le Programme canadien de fusion nucléaire : anatomie d'une décisionw, in Recherches sociographiques, vol. XXX, no 3, p. 421-446. 
chercheurs eux-mêmes, l'existence de deux listes de priorités contribue à renforcer leur autonomie en élargissant le nombre de domaines accessibles. De plus, les chercheurs peuvent invoquer la priorité accordée à leur domaine par un des deux niveaux de gouvernement pour contraindre l'autre à les appuyer financièrement. Tel a été le cas des chercheurs québécois en fusion nucléaire : tirant profit de la priorité que le gouvernement du Québec accordait à leur secteur, ils sont parvenus à obtenir l'engagement financier du gouvernement canadien qui $n$ 'avait pas intégré la fusion parmi ses priorités ${ }^{47}$.

Comme on peut le voir, il a été beaucoup question au cours des dernières années d'élaborer une politique scientifique et technologique propre à assurer la concentration de ressources dans un nombre limité de secteurs et de créneaux profitables au développement social et économique du Québec. Cette optique a permis à l'État québécois de se positionner comme intervenant privilégié dans le dossier de la science et de la technologie. Au cours des années 1970 et 1980, l'État s'est effectivement doté d'un ensemble d'instruments et d'organismes gouvernementaux susceptibles d'élaborer et d'appliquer une politique scientifique et technologique orientée sur le développement social ou économique du Québec. Depuis cette date, il existe au Québec un espace institutionnel où se négocie le degré d'autonomie du champ scientifique. Cette attitude interventionniste a conduit à l'expérimentation de plusieurs techniques permettant d'identifier et de choisir des priorités sectorielles. Dans toutes ces tentatives, la concertation a été un thème récurrent.

Dans la majorité des exercices que nous avons examinés, la démarche envisagée prévoyait que les représentants de l'industrie et des universités seraient étroitement associés au processus de décision. Malgré ces bonnes intentions, le rôle de choisir les priorités a toujours été assumé par un seul acteur : l'État. En maintes occasions, l'un ou l'autre des principaux intervenants $n$ 'a pas participé aux consultations : en 1987, les scientifiques $n^{\prime}$ ont pas été consultés par le ministère de l'Industrie et du Commerce; en 1982, les représentants de l'industrie n'ont pas participé directement au choix des priorités arrêtées dans $L e$ virage technologique. À la différence, par exemple, de ce qui se fait au Japon, on a utilisé une stratégie descendante («top-down») plutôt

47 Gingras, Y. et M. Trépanier (1989), op. cit. 
qu'une stratégie ascendante («bottom-up»). De plus, chaque fois que le gouvernement n'a pas fait appel aux utilisateurs de la R-D et aux producteurs, il a négligé soit les facteurs liés à la poussée scientifique et technologique, soit les facteurs relatifs à la traction du marché.

Le fait que les différents gouvernements aient pris les décisions sans consultation préalable ou en ne tenant pas compte des avis exprimés montre que l'apprentissage de ces nouvelles façons de gérer les activités scientifiques et technologiques s'est avéré difficile. Dans des sociétés comme le Japon et la Suède, grâce à une tradition bien établie de planification à long terme, de concertation et de consensus, la mise en place de ces mécanismes de gestion est naturelle. Au Québec, elle se heurte à plusieurs obstacles, notamment au fait que les politiques nationales sont souvent une réponse à des pressions exercées par différents groupes d'intérêts et qu'elles sont ainsi plutôt axées sur le très court terme $e^{48}$.

Sur un autre plan, les démarches gouvernementales ont manqué de crédibilité. À l'exception des travaux du Groupe de l'École Polytechnique, l'information sur laquelle étaient appuyés I'identification et le choix des secteurs prioritaires était sommaire et partielle. Tout en devant déterminer des secteurs avantageux pour le développement économique, les responsables des exercices n'ont pas fait usage de données précises sur l'évolution générale des besoins et des problèmes socio-économiques. Leur connaissance des forces et faiblesses du Québec sur ce plan est restée générale et approximative. Il en a été de même des données sur les forces et faiblesses du Québec au plan scientifique et technologique. Quant à la performance des chercheurs, à leur place sur la scène internationale et aux créneaux dans lesquels ils excellent, les responsables en avaient une connaissance impressionniste.

Malgré ces problèmes, le gouvernement a produit plusieurs listes de secteurs prioritaires. Dans la plupart des secteurs identifiés, des organismes de recherche et des activités de R-D ont

48 Pour plus de détails sur le Japon et la Suede, voir Martin, B. R. et J. Irvine (1989), op. cit., chapitres 6 et 9 . Sur le Canada et le Québec, on peut aussi consulter Martin, B. R. et J. Irvine (1989), op. cit., chapitre 8. Pour un exemple détaillé du processus décisionnel qui conduit à la promotion, au Québec et au Canada, de la fusion nucleaire au rang de priorité, voir Y. Gingras et M. Trépanier (1989), op. cit. 
été mis sur pied ou consolidés et on pourrait en conclure que les gouvernements fédéral et provincial ont planifié leurs investissements à partir de ces listes. On pourrait aussi en conclure qu'ils sont intervenus directement et massivement dans le choix des domaines et des objets de recherche sur lesquels les chercheurs font porter leurs efforts, réduisant d'autant l'autonomie du champ scientifique.

Une telle interprétation serait toutefois inexacte puisqu' on a aussi vu que le gouvernement du Québec a refusé, le plus souvent, de planifier ses efforts en fonction d'une liste de secteurs prioritaires précise, détaillée et bien définie. D'une part, le secteur ou le domaine dans lequel s'inscrit un projet de R-D n'a été que très rarement un critère d'évaluation. D'autre part, les listes de secteurs sont tellement larges et générales qu'à peu près tous les projets proposés peuvent $s^{\prime} y$ insérer sans trop de difficulté.

Ainsi, même si le gouvernement a pris des décisions visant à créer des équipes ou des centres de recherche dans des domaines qu'il juge prioritaires, on peut néanmoins dire que les chercheurs ont conservé pour une large part leur capacité à décider, en fonction des enjeux et intérêts propres au champ scientifique, les domaines et créneaux qui les intéressent. La majeure partie du soutien gouvernemental à l'activité scientifique et technique n'est pas liée à l'insertion d'un projet dans un des domaines ou créneaux d'une liste de priorités préétablie. Les chercheurs conservent ainsi une grande autonomie lorsque vient le temps d'orienter et de définir leurs activités de recherche. Dans leur étude sur le développement de la recherche en énergie solaire au Canada, Dalpé et Gingras arrivent aux mêmes conclusions. Leur analyse montre, en effet, que les chercheurs réussissent le plus souvent à poursuivre des travaux dans un domaine même en l'absence de programme prioritaire, ou encore à adapter leurs projets aux nouvelles priorités arrêtées par les gouvernements ${ }^{49}$.

Par ailleurs, même du strict point de vue de la planification de l'activité scientifique et technique en fonction d'objectifs de développement économique ou social, cette situation $n^{\prime}$ a pas que des désavantages. En effet, les caractéristiques spécifiques du Québec doivent inciter à la prudence celui qui s'engage dans une

49 Dalpé, Robert et Yves Gingras, «Recherche universitaire et priorités nationales : I'effet du financement public sur la recherche en énergie solaire au Canadan, in La Revue canadienne d'enseignement supérieur, 1990, vol. XX, no 2, p. 27-44. 
réflexion de ce type. Une petite société comme le Québec devra toujours importer des technologies et, pour être en mesure de s'approprier adéquatement les connaissances et les techniques développées ailleurs, elle devra conserver une base scientifique et technique suffisamment forte et diversifiée. De plus, pour bénéficier des percées réalisées à l'étranger, les chercheurs du Québec doivent payer leur droit d'entrée en apportant une contribution, fût-elle minime, à l'avancement des connaissances.

À l'exemple de pays comme la Suède, la Norvège et les Pays-Bas, le gouvernement du Québec a intérêt à tenir compte de cette situation quand il choisit des secteurs prioritaires et décide d'y concentrer une partie de ses ressources. Les gouvernements néerlandais et norvégien considèrent que l'investissement dans certains secteurs ne doit pas nuire au maintien d'un niveau élevé en recherche fondamentale. Ils voient dans ce type de recherche un moyen privilégié de conserver une base scientifique et technologique permettant à leur pays de s'approprier les connaissances produites ailleurs et de profiter des retombées économiques et sociales qui y sont liées. Autrement dit, il faut éviter que les efforts consentis dans les secteurs prioritaires empêchent de conserver un niveau minimal d'expertise dans les différents domaines scientifiques ${ }^{50}$.

II existe un autre danger pour le Québec à trop concentrer ses efforts sur des secteurs ou créneaux trop spécifiques ou trop différents de ce qui se fait dans les grands pays. Cela risque de provoquer un exode de scientifiques de valeur et donc d'appauvrir un potentiel où les ressources humaines sont rarement en surnombre ${ }^{51}$.

50 Pour les Pays-Bas, voir Ministerie van Onderwijs en Wetenschappen (1988), Towards a Science Policy for the 1990's, Zoetermeer, 4 juillet 1988; plus particulierement le chapitre 3 «Decision-making on knowledge: the political agenda for science policy in the Netherlands», p. 15-20. Pour la Norvege, voir Martin, B. R. et J. Irvine (1989), op. cit., p. 309-329.

51

OCDE (1991), Choisir les priorités scientifiques et technologiques, Paris, p. 66. 


\section{Conclusion}

Bien modestement, notre étude a donné un premier aperçu des efforts déployés par l'État québécois pour identifier et choisir, dans le cadre de sa politique scientifique et technologique, des domaines prioritaires où il allait concentrer ses investissements. Nous avons aussi voulu amorcer l'examen des effets qu'ont eus ces tentatives sur l'autonomie du champ scientifique.

II ressort de notre analyse que le gouvernement du Québec a la plupart du temps fonctionné en vase clos sans vraiment impliquer, dans la prise de décision et le choix des domaines prioritaires, les représentants des universités et de l'entreprise. Interventionniste, l'État québécois s'est doté d'un appareil de politique scientifique et, de concert avec cet appareil, les différents gouvernements au pouvoir sont intervenus pour mettre sur pied des activtés de R-D dans des secteurs qu'ils jugeaient importants. Curieusement, toutefois, nous avons constaté que ces interventions n'ont pas été assez nombreuses et extensives pour réduire significativement l'autonomie du champ scientifique : les chercheurs sont plutôt restés libres d'orienter leurs travaux, c'est-à-dire de choisir leurs objets de recherche, en fonction des enjeux et des intérêts propres à leur champ. En somme, l'interventionnisme de l'État n'a pas réellement quitté la sphère du discours politique.

Notre analyse ne permet pas de savoir comment et pourquoi les chercheurs sont parvenus à maintenir l'autonomie du champ scientifique à un degré élevé. À titre d'hypothèse, on peut cependant avancer, d'une part, que l'appareil de la politique scientifique mis en place par l'État québécois ne les a pas exclus des organismes et comités qui déterminent le financement de programmes ou de projets de R-D et, d'autre part, qu'ils sont encore en mesure d'exercer une influence décisive à ces positions stratégiques.

Le pouvoir législatif ne peut s'exercer directement sur la science sous peine de détruire, en ruinant l'autonomie du champ, ce qui garantit la perception sociale de son objectivité et de son impartialité. Les moyens d'intervention doivent donc maintenir l'illusion de la neutralité des personnes et des organismes qui les préparent $^{52}$. Pour assurer la légitimité de ses interventions,

52 Pollack, M. (1975), op. cit., p. 38. 
l'appareil de la politique scientifique accorde toujours une place aux chercheurs eux-mêmes. Dans ce contexte, si leur nombre ou leur prestige permet aux scientifiques d'imposer leurs propres critères d'évaluation, les décisions relèvent alors de l'évaluation par les pairs, et ils peuvent réussir à maintenir l'autonomie du champ à un niveau relativement élevé.

Pour examiner cette hypothèse, il faudra d'abord disposer de données sur le soutien financier accordé par l'État aux différents domaines ou créneaux de recherche afin de bien cerner le phénomène de concentration des ressources en fonction de secteurs prioritaires. Ensuite, il faudra s'intéresser à la composition des principaux organismes et comités ainsi qu'aux caractéristiques sociologiques des individus qui les composent. Cette deuxième étude, que nous ne faisons ici qu'évoquer, est très importante puisqu'elle permettra non seulement de préciser le degré d'autonomie du champ scientifique, mais aussi de se donner une meilleure compréhension du fonctionnement de la politique scientifique et de ses effets sur l'activité scientifique et technique. 